\title{
KRAS NP_004976.2:p.A146T
}

National Cancer Institute

\section{Source}

National Cancer Institute. KRAS NP 004976.2:p.A146T. NCI Thesaurus. Code C129524.

A change in the amino acid residue at position 146 in the GT Pase Kras protein where alanine has been replaced by threonine. 